\title{
Planning not to do something: Does intending not to do something activate associated sensory consequences?
}

\author{
SIMONE KÜHN \\ Ghent University, Ghent, Belgium \\ and Max Planck Institute for Human Cognitive and Brain Sciences, Leipzig, Germany \\ AND \\ MarCel Brass \\ Ghent University, Ghent, Belgium
}

\begin{abstract}
The present fMRI study investigated the central assumptions of ideomotor theory that actions become associated with their sensory consequences. Furthermore, we tested whether sensory effects can also become associated with the voluntary omission of an action. In a training phase, participants had to decide between executing an action and not executing it. Both decisions were followed by a specific effect tone. In the test phase, the participants had to carry out actions without hearing the effect tone. They either had to decide whether to execute an action or not or were instructed to execute an action or not. Our results reveal an increased activity in the auditory cortex elicited by responses that formerly elicited a tone-namely, self-chosen actions and selfchosen nonactions. Moreover, we found binding effects for stimulus-cued actions, but not for stimulus-cued nonactions. These findings support ideomotor theory by showing that a link exists between actions and their effects. Furthermore, our data demonstrate on a neural level that effect tones can become associated with intentionally not acting, therewith supporting the idea of a binding between the voluntary omission of an action and its effects in the environment.
\end{abstract}

Ideomotor theory assumes that we represent our behavior by anticipating its sensory consequences (Greenwald, 1970; James, 1890; Lotze, 1852; Prinz, 1997; Ziessler, Nattkemper, \& Frensch, 2004). Pianists, for example, imagine the melody that they want to play in order to make their fingers perform the necessary movements. From an ideomotor perspective, these motor representations result from learning the association between a motor command and its sensory consequences (action-effect). Such action-effect bindings are assumed to be bidirectional. In other words, perceiving the effect should trigger the associated movement, and carrying out the movement should activate the corresponding action effect. Experimentally, ideomotor theory has been tested in a number of behavioral studies using action-effect binding (Elsner \& Hommel, 2001; Hommel, 1996). In such experiments, participants usually learn a relationship between specific actions (e.g., pressing a right or a left key) and arbitrary sensory consequences (e.g., a high- or low-frequency tone) first. Then it is tested whether perceiving the sensory feedback (e.g., hearing a high-frequency tone) affects planning and execution of the congruent or the incongruent behavior. In accordance with ideomotor theory, these experiments demonstrate that perceiving congruent sensory feedback facilitates response selection, whereas perceiving incongruent sensory feedback interferes with response selection.

Another way to investigate the assumption of bidirectional links between an action and the associated sensory consequence is to test whether executing such actions activates neural structures that are involved in the processing of the sensory feedback. In other words, does executing an action that has been consistently followed by a tone activate the auditory cortex? One aim of the present study was to investigate this question, using functional MRI.

The second aim of the study was to investigate the minimal conditions under which actions become associated with their sensory effects? This point relates to the formation of action-effect bindings. One could argue that effects can become associated only with the actual motor command. If this holds true, one would expect that action-effect bindings are formed only when participants actually execute responses followed by sensory effects. However, it has recently been argued that participants do not necessarily need to execute responses but that motor preparation is sufficient to create the minimal conditions for the formation of action-effect bindings (Ziessler \& Nattkemper, 2002). Going even one step further, recent

S. Kühn, simone.kuhn@ugent.be 
findings indicate that effects can also become associated with the decision not to execute an action (nonactioneffect bindings; Kühn, Elsner, Prinz, \& Brass, 2009). In the present study, we wanted to test whether executing nonactions that have been associated with an effect tone would activate the auditory cortex. The third aim of the study was to investigate the conditions under which action/ nonaction-effect bindings are retrieved. Here, we compared self-chosen and stimulus-cued actions/nonactions in the retrieval phase. The crucial question was whether action/nonaction-effect bindings that have been learned for self-chosen actions activate the sensory consequence when participants have to carry them out in response to a stimulus in the test phase.

We exposed participants to an acquisition phase outside the scanner in which self-chosen actions and self-chosen nonactions led to two different effect tones. In the subsequent scanning session, all effect tones were omitted, and the participants were asked to decide between acting and not acting or were cued by a stimulus to act or not to act. We predicted - in line with our behavioral findings - binding effects for self-chosen actions, as well as for self-chosen nonactions. These binding effects should be reflected in increased auditory cortex activation. Furthermore, we expected to find activation of the auditory cortex in the condition in which the participants were cued by a stimulus to execute an action as well, because the preparation and execution of the response could make use of the activation of the sensory consequence as well. Importantly, because stimulus-cued nonactions do not involve any decision not to execute an action, we expected no effect anticipation for stimulus-cued nonactions.

\section{METHOD}

\section{Participants}

We recruited 14 healthy volunteers ( 8 women and 6 men; age: $M=22.6$ years, ranging from 19 to 29 ) from whom we obtained written consent prior to the scanning session. All the participants had normal or corrected-to-normal vision. No participant had a history of neurological, major medical, or psychiatric disorder. All the participants were right-handed, as assessed by a handedness questionnaire (van Strien, 1992; mean score $=9.6$ ).

\section{Behavioral Task}

The experimental procedure consisted of two phases: an acquisition phase administered outside the MR scanner and another task similar to that in the acquisition phase - namely, the action phaseadministered during fMRI measurement (see Figure 1). In the acquisition phase, the participants performed a two-choice reaction task. The trials started with a jitter of $0,500,1,000$, or $1,500 \mathrm{msec}$ to obtain an interpolated temporal resolution of $500 \mathrm{msec}$. Then a fixation cross was presented at the center of the screen for $500 \mathrm{msec}$. After a blank of $100 \mathrm{msec}$, a two-colored (blue-orange) circle was presented to indicate that the participants should choose between pressing two buttons with their right and left index fingers simultaneously (self-chosen action, self-go) or refrain from pressing both buttons (self-chosen nonaction, self-no-go). We chose bilateral responses in order to avoid strong lateralization of motor-related brain activity and in order to make the results comparable to those in Kühn, Elsner, Prinz, and Brass (2009). The participants were asked to aim at an equal distribution of responding and not responding without having a certain rule in mind. When a response was given, we presented a tone $250 \mathrm{msec}$ after the response. For no-buttonpress trials, we determined the interval before tone presentation with reference to the history of reaction times (RTs) on response trials. This interval allowed a certain variance in response RTs but still, as the participants reported in the debriefing, suggested the feeling of causation of the tone after the decision not to respond. The tones used were $400-, 600-$, and $800-\mathrm{Hz}$ sinusodial wave tones of 200 -msec duration

\section{Acquisition Phase}

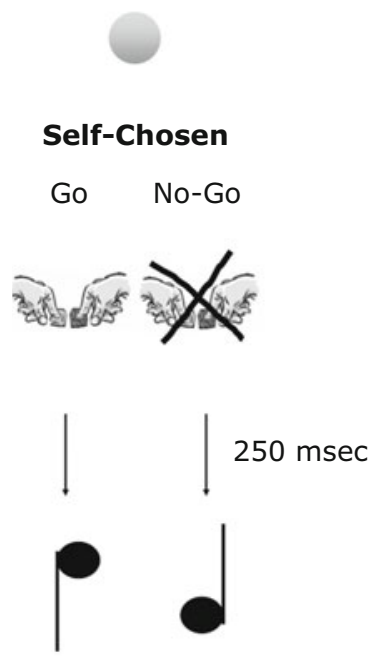

Action Phase

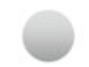

Self-Chosen

Stimulus Cued

Go

No-Go Go No-Go

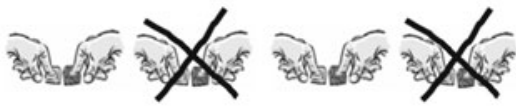

Figure 1. Schematic drawing of the conditions in the experiment. 
delivered via headphones. The action-effect mapping was balanced across participants, and we selected two of the three tones that were associated with responding or the choice not to respond. The acquisition phase outside the scanner consisted of 480 trials.

Inside the scanner, the participants performed a task similar to that in the acquisition phase. The main difference was that no effect tones were presented. In addition, we presented blue and orange squares indicating that the participants had to press both buttons (stimulus-cued action, stimulus-go) or were not allowed to press the buttons (stimulus-cued nonaction, stimulus-no-go). The two blocks consisted of 96 trials each, comprising 48 voluntary trials and 24 stimulus-cued action and 24 stimulus-cued nonaction trials.

In a subsequent tone phase, we presented the two effect tones and a third tone (odd tone) that was different from the effect tones, to which the participants had to respond. The tone phase consisted of two blocks of 128 trials each, comprising 32 presentations of each of the three different tones and 32 null events, which were pseudorandomly interspersed. Overall, the experiment in the scanner lasted about $45 \mathrm{~min}$.

\section{Scanning Procedure}

Images were collected with a 3T Magnetom Trio MRI scanner system (Siemens Medical Systems, Erlangen, Germany) using an 8-channel radio frequency head coil. First, high-resolution anatomical images were acquired using a T1-weighted 3D MPRAGE sequence $(\mathrm{TR}=2,530 \mathrm{msec}, \mathrm{TE}=2.58 \mathrm{msec}, \mathrm{TI}=1,100 \mathrm{msec}$, acquisition matrix $=256 \times 256 \times 176$, sagittal FOV $=220 \mathrm{~mm}$, flip angle $=7^{\circ}$, voxel size $=0.86 \times 0.86 \times 0.9 \mathrm{~mm}^{3}$ ). Wholebrain functional images were collected using a T2*-weighted EPI sequence sensitive to BOLD contrast $(\mathrm{TR}=2,000 \mathrm{msec}, \mathrm{TE}=$ $35 \mathrm{msec}$, image matrix $=64 \times 64, \mathrm{FOV}=224 \mathrm{~mm}$, flip angle $=$ $80^{\circ}$, slice thickness $=3.0 \mathrm{~mm}$, distance factor $=17 \%$, voxel size $=$ $3.5 \times 3.5 \times 3 \mathrm{~mm}^{3}, 30$ axial slices). On average, 247 image volumes were acquired per action run all aligned to AC-PC.

\section{fMRI Data Preprocessing and Main Analysis}

The fMRI data were analyzed using the SPM5 software (Wellcome Department of Cognitive Neurology, London). The first four volumes of all EPI series were excluded from the analysis to allow the magnetization to approach a dynamic equilibrium. Data processing started with slice time correction and realignment of the EPI data sets. A mean image for all EPI volumes was created, to which individual volumes were spatially realigned by means of rigid body transformations. The high-resolution structural image was coregistered with the mean image of the EPI series. Then the structural image was normalized to the Montreal Neurological Institute (MNI) template, and the normalization parameters were applied to the EPI images to ensure an anatomically informed normalization. During normalization, the anatomy image volumes were regridded to $1 \times$ $1 \times 1 \mathrm{~mm}^{3}$. A commonly applied filter of $8-\mathrm{mm}$ full-width at half maximum was used. Low-frequency drifts in the time domain were removed by modeling the time series for each voxel by a set of discrete cosine functions to which a cutoff of $128 \mathrm{sec}$ was applied. The subject-level statistical analyses were performed using the general linear model. The main events of interest were the periods after the onset of the stimulus in the action phase. Vectors containing the event onsets were convolved with the canonical haemodynamic response function (HRF) to form the main regressors in the design matrix (the regression model). The vectors were also convolved with the temporal derivatives, and the resulting vectors were entered into the model. The statistical parameter estimates were computed separately for each voxel for all columns in the design matrix. Contrast images were constructed from each individual to compare the relevant parameter estimates for the regressors containing the canonical HRF. The group-level random effects analysis was then performed. A onesample $t$ test was performed for each voxel of the contrast images. In order to test the predictions of our study, we looked for an interaction of the self-chosen/stimulus-cued and go/no-go factors in the auditory cortex. In order to confirm that the activation in the auditory cortex was indeed active during tone perception, we computed the contrast of action-associated tone and nonaction-associated tone versus null events. To explore multimodal brain areas that might potentially be involved in binding, we computed three contrasts: self-chosen-go $>$ stimulus-cued-no-go, self-chosen-no-go $>$ stimulus-cued-no-go, and stimulus-cued-go $>$ stimulus-cued-no-go. Then we looked at the overlap of the three contrasts by multiplying the resulting thresholded tmaps with one another. The resulting statistical values were thresholded at $p<.001(z>3.09$, uncorrected). A significant effect in the interaction analysis was reported when the volume of the cluster was greater than the Monte Carlo simulation-determined minimum cluster size within the temporal lobe ( $>6$ voxels), above which the probability of a Type I error was $<.05$ (AlphaSim; Ward, 2000). All other whole-brain contrasts were thresholded (at $p<.001$ ) with a cluster size of $>22$ voxels, which resulted from a Monte Carlo simulation on the whole-brain volume. The maps were overlaid onto a normalized T1-weighted MNI single-subject template (colin27).

For the signal change analysis, we defined a region of interest (ROI) consisting of the peak voxel in the left auditory cortex resulting from the whole-brain interaction contrast of (stimulus-cuedgo $>$ stimulus-cued-no-go) $>$ (self-chosen-go $>$ self-chosenno-go). For each participant, region, and condition, the mean percentage of signal change over a time window of 4-6 sec after stimulus onset was calculated separately, using MarsBaR (Brett, Anton, Valabregue, \& Poline, 2002) and was compared by means of paired $t$ tests. The percentage of signal change is calculated as the change from the mean signal in the ROI over the whole time course.

\section{RESULTS}

\section{Behavioral Data}

The amount of self-chosen actions and self-chosen nonactions throughout the whole experiment was not significantly different, either in the acquisition phase outside the scanner $[50.9 \%$ go, $49.1 \%$ no-go; $t(13)=0.82, p=$ $.43]$ or in the action phase inside the scanner $(51.5 \%$ go, $48.5 \%$ no-go; $t(12)=1.22, p=.25]$. In the stimulus-cued condition during the action phase, the participants made, on average, $0.03 \%$ errors by either omitting a response although they had to respond or giving a response although they were not allowed to. Those trials were excluded from further analyses.

\section{fMRI Data}

The most straightforward way to test our prediction is to look for an interaction of the factors self-chosen/ stimulus-cued and go/no-go in the auditory cortex. More specifically, we predicted more auditory activation in all conditions than in the stimulus-cued nonaction condition, for which we predicted less activation in the auditory cortex. In accordance with this prediction, a significant interaction in the left auditory cortex (BA 22) was found (see Table 1). An ROI analysis in the left auditory cortex revealed an increase in auditory cortex activity in response to self-chosen actions, self-chosen nonactions, and stimulus-cued actions, and less auditory activation was found for stimulus-cued nonactions (see Figure 2A). A repeated measures ANOVA on percentage of signal changes with the factors self-chosen/stimulus-cued and go/no-go revealed no significant main effects of selfchosen/stimulus-cued $[F(1,13)=0.92, p>.05]$ but a significant effect of go/no-go $[F(1,13)=9.54, p<.01]$ and a significant interaction $[F(1,13)=29.72, p<.001]$. 
Table 1

MNI Coordinates of the Interaction of the Self-Chosen/Stimulus-Cued and Go/No-Go Factors in the Auditory Cortex $(p<.001$, Cluster $>6$ Voxels Based on Monte Carlo Simulations on the Temporal Lobe)

\begin{tabular}{ccccc}
\hline Area & BA & Peak Coordinates (MNI) & $z$ Score & Voxel Extent \\
\hline Left auditory cortex & 22 & $-56,4,0$ & 3.60 & 7 \\
\hline
\end{tabular}

Paired $t$ tests revealed significant differences between the reactivation in self-chosen action and stimulus-cued nonaction $[t(13)=2.66, p<.05]$, self-chosen and stimuluscued nonaction $[t(13)=2.38, p<.05]$, and stimulus-cued action and stimulus-cued nonaction $[t(13)=4.75, p<$ $.001]$. In order to confirm that the activation in the auditory cortex was indeed active during tone perception, we compared the auditory cortex activation found in the inter- action analysis during the action phase with activation in the tone phase from the contrast "action-associated tone" and "non-action-associated tone" versus null events and found considerable overlap ( 5 of 7 voxels) between both (see Figure 2A, Table 2).

To explore the multimodal areas that could be involved in the binding process, we computed three contrasts comparing each condition that showed increased auditory cor-

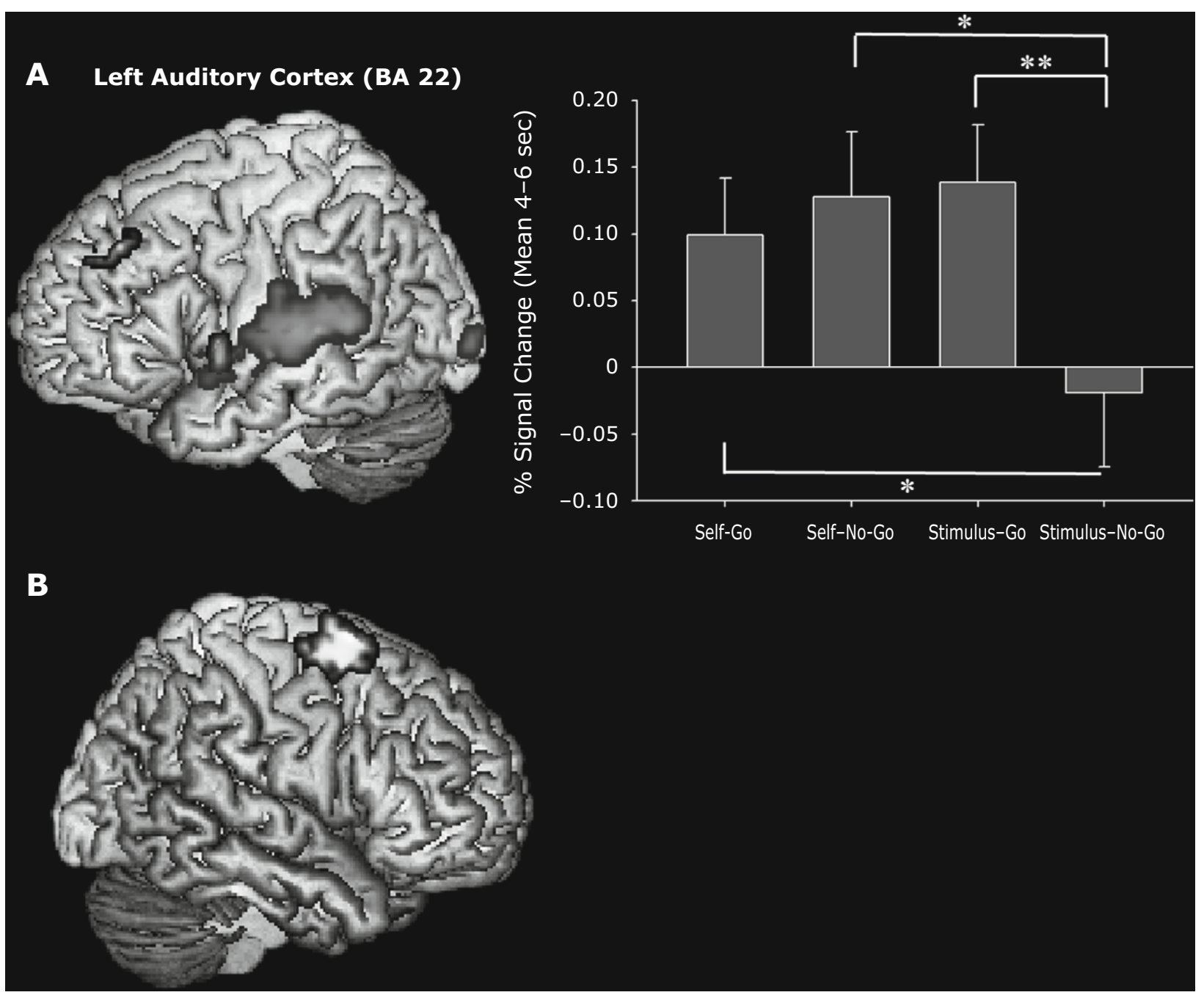

Figure 2. (A) Significant brain areas of the interaction (stimulus-cued-go $>$ stimulus-cued-no-go) $>$ (self-chosen-go $>$ self-chosenno-go) and percentages of signal changes for a region of interest in the left auditory cortex, depicted in orange (dark gray in printed version). In light blue (light gray in printed version), the contrast of action-associated tone and non-action-associated tone versus null events in the tone phase is depicted. The activation in the dorsolateral prefrontal cortex does not survive the Monte Carlo simulation that was restricted to the temporal lobe on the basis of an a priori hypothesis. (B) Overlap between three contrasts-self-chosen-go $>$ stimulus-cued-no-go; self-chosen-no-go $>$ stimulus-cued-no-go; stimulus-cued-go $>$ stimulus-cued-no-go-in the right dorsal premotor cortex (BA 6). Self, self-chosen; stimulus, stimulus cued. ${ }^{*} p<.05 .{ }^{* *} p<.01$, error bars depicting the standard error of the mean. 
Table 2

MNI Coordinates of the "Action-Associated Tone" and "Non-Action-Associated Tone" Versus Null Events Contrast $(p<.001$, Cluster $>22$ Voxels Based on Monte Carlo Simulations on Whole-Brain Volume)

\begin{tabular}{lcccc}
\hline \multicolumn{1}{c}{ Area } & BA & Peak Coordinates (MNI) & $z$ Score & Voxel Extent \\
\hline Left auditory cortex & 22 & $-53,-35,7$ & 5.12 & 319 \\
Left visual cortex & 18 & $-7,-81,21$ & 4.65 & 348 \\
Right auditory cortex & 22 & $67,-21,14$ & 4.52 & 234 \\
Left putamen & & $-25,11,4$ & 4.43 & 52 \\
Right dorsolateral prefrontal cortex & 46 & $39,46,35$ & 4.31 & 51 \\
Right putamen & & $28,11,7$ & 3.84 & 50 \\
\hline
\end{tabular}

tex activation and behavioral binding effects in our previous behavioral study (Kühn, Elsner, et al., 2009) with the instructed nonaction condition that did not show binding effects (self-chosen-go $>$ stimulus-cued-no-go; selfchosen-no-go $>$ stimulus-cued-no-go; stimulus-cuedgo $>$ stimulus-cued-no-go). Overlap between the three contrasts was found in the right dorsal premotor cortex (dPMC) (see Figure 2B).

\section{DISCUSSION}

According to ideomotor theory, actions are voluntarily produced by anticipating their effects once an association between a specific behavior and its perceptual consequences has been established (Greenwald, 1970). With our present study, we show that executing an action that has been associated with an effect tone activates brain areas that are involved in the processing of its auditory effect. This auditory cortex activation occurs regardless of whether the action is cued by a stimulus or is self-chosen. Moreover, we also show auditory cortex activation for the sensory consequences of self-chosen nonactions. Importantly, less auditory cortex activation was found for stimulus-cued nonactions. Our findings support the idea that actions become associated with their sensory consequences. Most important, the present results show that sensory consequences can also become associated with the decision not to execute an action. This clearly demonstrates that the formation of effect bindings does not necessarily rely on the activation of a motor command. However, the sensory consequence of a nonaction is retrieved only when participants choose not to act, suggesting that the choice component is crucial to retrieving nonaction effects, but not to retrieving action effects.

\section{Action-Effect Binding: Evidence for Direct Action-Effect Links}

The assumption that actions become associated with their sensory consequences has so far primarily been tested with paradigms that investigated whether perceiving the effect of an action activates the corresponding motor representation (Elsner \& Hommel, 2001; Kunde, 2004). In the preset study, we used a different logic to investigate this question. We argued that when actions become associated with a specific sensory consequence in a training phase, they should activate the brain areas that are involved in the processing of this sensory consequence in a later test phase. The present findings clearly support this pre- diction from ideomotor theory. However, because of the low time resolution of fMRI, we cannot be sure that sensory activation indeed precedes motor execution, as would be predicted from ideomotor theory. But given previous behavioral results, it seems very likely that the sensory consequence of an action becomes part of the motor representation and, therefore, precedes motor execution. As was outlined above, previous findings have shown that presenting the sensory consequence of an action facilitates motor execution of that action, suggesting that the sensory consequence is part of the motor representation. Moreover, there is evidence in favor of the notion that the sensory consequences of actions are anticipated during the action preparation phase (Kühn, Keizer, Rombouts, \& Hommel, 2011). During the preparation of manual actions, we found activation of the extrastriate body area that is known to mediate the perception of body parts, whereas facial actions were preceded by activation of the fusiform face area, known to mediate face perception. The present findings extend these observations to nonactions. Moreover, they show that the anticipatory activation can also be shown when action effects are experimentally manipulated and acquired only during a short training phase, as compared with action effects that have been established over a lifetime, as in Kühn et al. (2011). However, in order to definitively show that areas involved in sensory processing already become activated before the response is executed, it is crucial to replicate our experiment with a method that has a better time resolution, such as EEG or MEG.

However, there is another point that needs further clarification. If the sensory effect is part of the motor representation, one would expect to find multimodal areas, such as the premotor or parietal cortex, to be active, in addition to primary sensory brain areas. In the interaction contrast, however, we did not observe premotor or parietal brain activity. We assume that in a situation in which action effects have been recently acquired, activation in primary sensory brain areas might be stronger than activation in multimodal brain areas. Furthermore, the lack of premotor or parietal cortex activation might be due to a lack of statistical power in the interaction contrast. And indeed, when exploring the overlap of the contrasts of the conditions that do show binding effects (higher activity in the auditory cortex) and the condition that does not show a binding effect - namely, the stimulus-cued-no-go condition - we find overlap in the right $\mathrm{APMC}$, a region that has been related to motor preparation (e.g., Picard \& Strick, 2001) and stimulusguided behavior (Goldberg, 1985). 


\section{Formation of Nonaction-Effect Bindings}

Another crucial finding of the present study relates to the observation of nonaction-effect bindings. This finding supports previous behavioral research suggesting that participants associate sensory effects with the decision not to execute an action. In our opinion, this finding has important theoretical implications by demonstrating that effect bindings can be formed without involving a motor command. But what exactly becomes associated in the case of nonaction-effect bindings? One possibility is that effects become associated with a representation of the decision. Alternatively, one could argue that nonaction involves, to some degree, motor preparation. In a previous study, we could demonstrate that the only brain area that shows stronger activation for a self-chosen action, as compared with self-chosen nonaction, is the primary motor cortex (Kühn \& Brass, 2009). Although this finding clearly indicates that nonactions do not involve the activation of a motor command, it is consistent with the idea that self-chosen actions and nonactions involve motor preparation and that the sensory consequence becomes associated with a motor representation that is related to motor preparation.

\section{Retrieval of Nonaction-Effect Bindings: Stimulus-Cued Versus Self-Chosen}

One of the most crucial findings of the present study, however, is the dissociation in effect anticipation for stimuluscued and self-chosen nonactions. Whereas self-chosen nonactions showed the same kind of effect anticipation in the retrieval phase as self-chosen actions or stimulus-cued actions, stimulus-cued nonactions did not show such an anticipation effect. This finding is consistent with a similar dissociation of self-chosen nonaction and stimulus-cued nonaction in a previous behavioral experiment (Kühn, Elsner, et al., 2009). In this study, we demonstrated that effect bindings were formed for self-chosen actions, selfchosen nonactions, and stimulus-cued actions, but not for stimulus-cued nonactions. Furthermore, this dissociation is in accordance with previous neurophysiological data showing P2 and N2 similarities between self-chosen actions, self-chosen nonactions, and stimulus-guided actions but a clear difference for stimulus-cued nonactions (Kühn, Gevers, \& Brass, 2009). The failure to retrieve sensory effects of nonactions when they were stimulus cued reveals that the decision process is crucial to retrieving nonaction effects, but not to retrieving action effects.

\section{Conclusions}

To summarize, our results show that self-chosen actions involve an anticipation of the sensory effects in the environment. Furthermore, our data suggest that sensory effects can become associated with a choice not to act, supporting the idea that the activation of a motor command is not required in the formation of effect bindings. Finally, our data suggest that nonaction-effect bindings are retrieved only when the nonaction is self-chosen, but not when it is stimulus cued.

\section{AUTHOR NOTE}

The first author is a Postdoctoral Fellow of the Research Foundation Flanders (FWO). The research was funded by a special research fund (BOF, University of Ghent). Correspondence concerning this article should be addressed to S. Kühn, Faculty of Psychology and Educational Sciences, Department of Experimental Psychology and Ghent Institute for Functional and Metabolic Imaging, Henri Dunatlaan 2, 9000 Ghent, Belgium (e-mail: simone.kuhn@ugent.be).

\section{REFERENCES}

Brett, M., Anton, J.-C., Valabregue, R., \& Poline, J.-B. (2002). Region of interest analysis using an SPM toolbox [Abstract]. NeuroImage, 16(2, Suppl. 1).

Elsner, B., \& Hommel, B. (2001). Effect of anticipation and action control. Journal of Experimental Psychology: Human Perception \& Performance, 27, 229-240.

GOLDBERG, G. (1985). Supplementary motor area structure and function: Review and hypothesis. Behavioral \& Brain Sciences, 8, 567-616.

Greenwald, A. G. (1970). Sensory feedback mechanisms in performance control: With special reference to the ideo-motor mechanism. Psychological Review, 77, 73-99.

Hommel, B. (1996). The cognitive representation of action: Automatic integration of perceived action effects. Psychological Research, 59, 176-186.

JAMES, W. (1890). The principles of psychology. New York: Dover.

KÜHN, S., \& BRASS, M. (2009). When doing nothing is an option: The neural correlates of deciding whether to act or not. NeuroImage, $\mathbf{4 6}$, 1187-1193.

Kühn, S., Elsner, B., Prinz, W., \& Brass, M. (2009). Busy doing nothing: Evidence for nonaction-effect binding. Psychonomic Bulletin \& Review, 16, 542-549.

KüHn, S., Gevers, W., \& Brass, M. (2009). The neural correlates of intending not to do something. Journal of Neurophysiology, 101, 1913-1920.

Kühn, S., Keizer, A., Rombouts, S. A. R. B., \& Hommel, B. (2011). The functional and neural mechanisms of action preparation: Roles of EBA and FFA in voluntary action control. Journal of Cognitive Neuroscience, 23, 214-220.

Kunde, W. (2004). Response priming by supraliminal and subliminal action effects. Psychological Research, 68, 91-96.

Lotze, R. H. (1852). Medicinische Psychologie oder Physiologie der Seele [Medical psychology or physiology of the soul]. Leipzig: Weidmann'sche Buchhandlung.

PICARD, N., \& STRICK, P. L. (2001). Imaging the premotor areas. Current Opinion in Neurobiology, 11, 663-672.

PrINZ, W. (1997). Perception and action planning. European Journal of Cognitive Psychology, 9, 129-154.

van Strien, J. W. (1992). Classificatie van links- en rechtshandige proefpersonen [Classification of left- and right-handed test subjects]. Nederlandes Tijschrift voor de Psychologie en Haar Grensgebieden, 47, 88-92.

WARD, B. D. (2000). Simultaneous inference for fMRI data. AFNI AlphaSim Documentation. Milwaukee: Medical College of Wisconsin.

Ziessler, M., \& NATTKEMPER, D. (2002). Effect anticipation in action planning. In W. Prinz \& B. Hommel (Eds.), Common mechanisms in perception and action: Attention and performance XIX (pp. 645-672). Oxford: Oxford University Press.

Ziessler, M., NATTKEMPER, D., \& Frensch, P. A. (2004). The role of anticipation and intention in the learning of effects of self-performed action. Psychological Research, 68, 163-175.

(Manuscript received September 12, 2009; revision accepted for publication July 11, 2010.) 